\title{
Analytical modelling of spiral cantilever structure for vibration energy harvesting applications
}

\author{
Nevin Augustine $^{1 *}$, Hemanth Kotturu ${ }^{2}$, S. Meenatchi Sundaram ${ }^{3}$, G.S. Vijay ${ }^{4}$ \\ ${ }^{1}$ Dept. of Instrumentation and Control Engineering, Manipal Institute of Technology, Manipal Academy of Higher Education \\ ${ }^{2}$ Dept. of Instrumentation and Control Engineering, Manipal Institute of Technology, Manipal Academy of Higher Education \\ ${ }^{3}$ Dept. of Instrumentation and Control Engineering, Manipal Institute of Technology, Manipal Academy of Higher Education \\ ${ }^{4}$ Dept. of Mechanical and Manufacturing Engineering, Manipal Institute of Technology, Manipal Academy of Higher Education \\ *Corresponding author E-mail:nevin.augustine@manipal.edu
}

\begin{abstract}
Research on harvesting energy from natural resources is more focused as it can make microelectronic devices self-powered. MEMS based vibration energy harvesters are gaining its popularity in recent days to extract energy from vibrating objects and to use that energy to power the sensors. A solution for the major constrain for vibration energy harvesting in micro scale has been addressed in this paper. Cantilever beams coated with piezoelectric materials which are optimized to resonate at the source vibration frequency are used in most of the traditional vibration energy harvesting applications. In micro scale such structures have very high natural frequency compared to the ambient vibration frequencies due to which frequency matching is a constrain. Tip mass at the end of the cantilever reduces the resonant frequency to a great extent but adds to complexity and fabrication difficulties. Here, we propose a spiral geometry for micro harvester structures with low fundamental frequencies compared to traditional cantilevers. The spiral geometry is proposed, simulated and analyzed, to show that such a structure would be able to vibrate near resonance at micro scale. The analysis consists of Modal analysis, Mises stress analysis and displacement analysis in COMSOL Multiphysics. The result shows that the frequency has been reduced by a factor of 300 when compared to normal cantilever in the same volume. The work provides guideline for vibration energy harvesting structure design for an improved performance.
\end{abstract}

Index Terms: Vibration energy harvesters, MEMS, modal analysis, mises stress analysis, spiral geometry.

\section{Introduction}

Wireless technology and wireless systems have become part of everyday life today. Portable devices, computer peripherals etc. are examples of such systems. The advantage of wireless systems are predominant in improved felxibilty, ease of placing sensor nodes etc. At present, many wireless sensor nodes are battery powered and operate on an extremely economical energy budget since continuous battery replacement is not an option for networks with thousands of physically embedded nodes[1]. Some specific examples of wireless sensor networks include the WiseNET platform developed by the Swiss Centre for Electronics and Micro technology (CSEM) [3] and those discussed by Warneke et al [4] and Callahan [5].

The low-power wireless sensor nodes provide a real incentive for investigating alternative types of power source to traditional batteries. The subject of this paper is design of kinetic energy generators, which convert energy in the form of mechanical movement present in the application environment into electrical energy. Kinetic energy is typically present in the form of vibrations, random displacements or forces and is typically converted into electrical energy using electromagnetic, piezoelectric or electrostatic mechanisms. Suitable vibrations can be found in numerous applications including common household goods, industrial plant equipment, moving structures such as automobiles and aero planes and structures such as buildings and bridges [6]. Human based applications are characterized by low frequency high amplitude displacements [7,8].The amount of energy generated by this approach depends fundamentally upon the quantity and form of the kinetic energy available and the efficiency of the generator and the power conversion electronics.

The most common and widely used structure for vibration harvesting is cantilever beams with tip mass connected to them. The efficiency of such devices is mainly depends on the natural frequency of the device. For transferring maximum energy from the source of vibration to the harvesting device, it is important that the frequency of vibration matches the natural frequency of the device. But as devices are fabricated in micro or near micro sizes, the natural frequency of the device becomes extremely high in range of few kHz's. But all ambient vibrations have very low frequency band in range of few hundred Hz. Thus it is inevitable to narrow down the natural frequency of the devices for greater efficiency. A promising structure that has been discussed in literature is spiral and buckled structures which can reduce the natural frequency of vibration to a much lower range [9]. We propose a much simpler structure of square shaped cantilever beam with tangential displacement at the tip of a spiral several times that of an equivalent length straight ceramic strip for energy harvesting applications. This paper discusses on the simulation studies of spiral structures as prominent substitute for normal cantilevers for increasing the efficiency of the devices. The structure has been simulated with different number of spiral beams from 8-20 beams and structural analysis has been performed on each structures. Effect of proof mass of various dimensions is also analyzed by finite element analysis. Different transduction mechanisms can be implemented in this structure for vibration 
energy harvesting applications.

\section{Structure design}

The schematic of the Spiral cantilever beam is shown in Figure.1, which consists of square shaped spiral spring, sharing the same anchor in the center of the device within an area of $100 \mu \mathrm{mX} 100 \mu \mathrm{m}$. A large proof mass of $10 \mu \mathrm{m}$ cube is mounted beneath the free end, therefore, the natural frequency was effectively reduced to match the environmental vibrations. With sufficient out of plane (z-axis) stiffness, the spiral structure will only vibrate in plane. In a practical device, top and bottom wafers will further constrain motion in the thickness direction under extreme conditions.

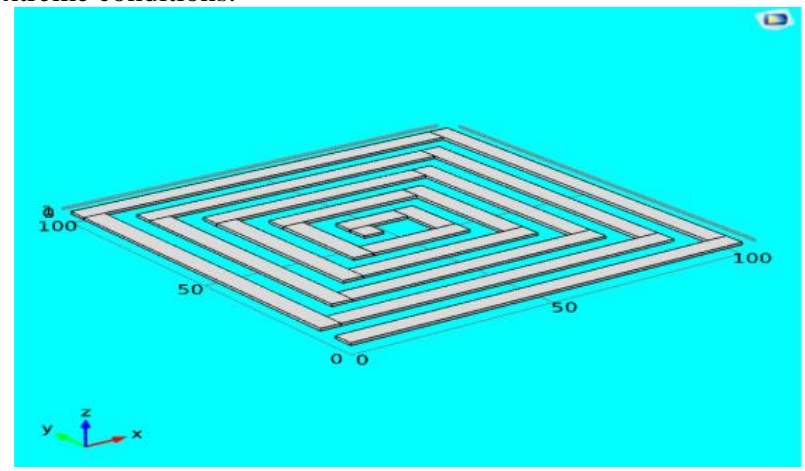

Fig. 1: Spiral cantilever structure

The spiral-shaped beam, is fixed at one end and acts as a cantilever structure with tip mass of a high density material. Here, the spiral cantilever is modeled as a series of beam segments with the same width and thickness. Each beam segment is connected to the adjacent beams at its two ends; both the bending motion and torsion of each beam segment will result in the next beam move out of the main plane. The governing equations of bending and torsion for the free vibration of each beam segment (with damping neglected) can be written according to Euler-Bernoulli beam bending equation as:

$$
y I\left[\frac{d^{4}(w i)}{d x^{4}}\right]+P A\left[\frac{d^{2}(w i)}{d t^{2}}\right]=0
$$

where $\mathrm{t}$ is the time, $\mathrm{x}$ is the axis along the length of the beam, YI is the bending stiffness, $\mathrm{Y}$ is the elasticity modulus, I is the moment of inertia, $\rho \mathrm{A}$ is the mass per unit of length, $\mathrm{A}$ is the area of the beam section, $\rho$ is the density, and wi is the out-of plane displacement of each beam in the OSS. Using separation of variables, the solution can be found as multiple functions in space and time. Using this method means that all of the structure is moving according to the same time function (phase). Thus, the displacement of any position of a beam along the time can be evaluated by separation of variables

$$
\text { wi }(x, t)=W_{i}(x) M(t)
$$

where Wi ( $\mathrm{x})$ is the displacement function (mode shape) and $\eta(\mathrm{t})$ is the time function. The general solution for equation (1) considering equation (2) is:

$$
W(x)=\sum_{j=1}^{4} A_{i j} e^{s i j x}
$$

\section{Analyses of different structures}

Spiral structures with different number of beams within the same cross sectional area have been simulated and the Finite element analysis on different parameters have been performed. The spiral structure with 20 beams was simulated and an Eigen frequency of $12.7 \mathrm{KHz}$ was obtained (Fig.2). Tip mass was made of highly density MEMS compatible copper material which could further reduce the natural frequency of the device. Modal analysis of the structure was performed to determine the resonant frequencies and vibration modes. The dimensions of the spiral cantilever and the proof mass were adjusted to yield in-plane working modes. The finally determined spiral spring has 20 interconnected gave a resonant frequency of $10.5 \mathrm{KHz}$ (Figure.3). The lowest 3 natural frequencies were evaluated at $10.5 \mathrm{KHz}, 11.4 \mathrm{KHz}$, and $14.5 \mathrm{KHz}$. The corresponding vibration modes are all purely in-plane. Stress analysis of the structures have been simultaneously carried out so the piezoelectric strips can be placed on corresponding areas of maximum stresses for effective conversion of mechanical energy to electrical energy. The result is shown in figure. 4

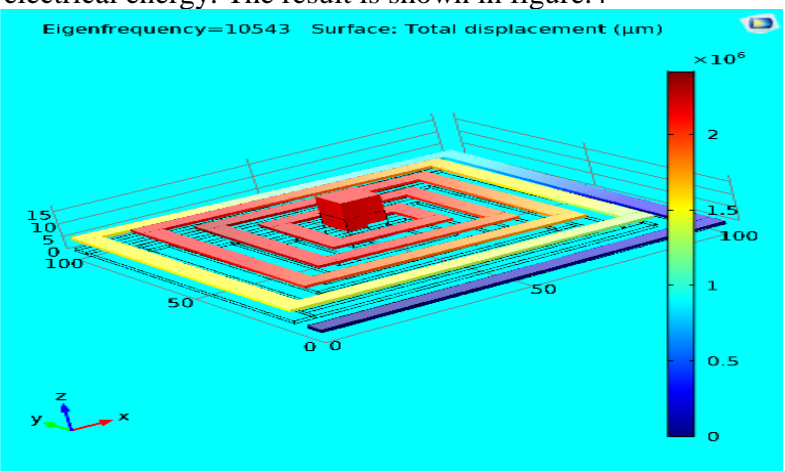

Fig. 2: Frequency analysis of 20 beam structure

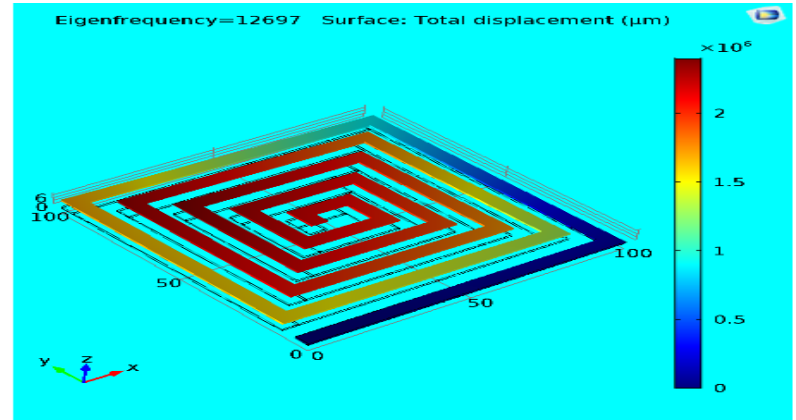

Fig. 3: Simulation of 20 beam structure with tip mass

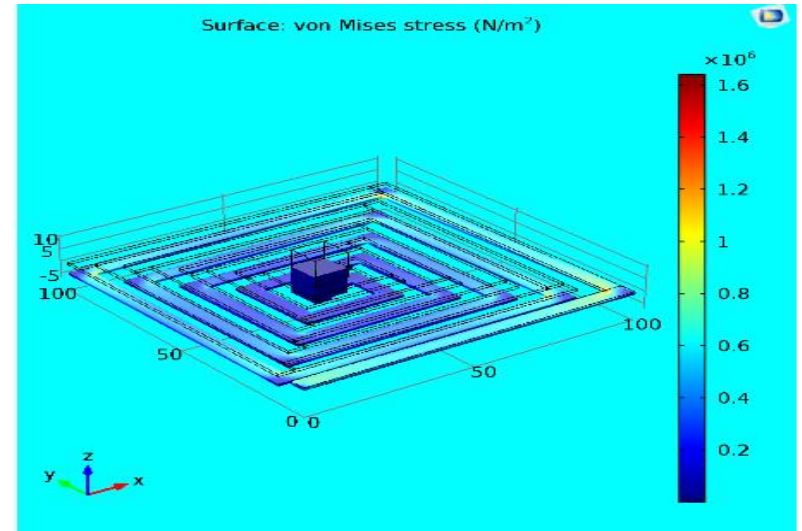

Fig. 4: Mises stress analysis plot

\section{Analytical modelling/dynamic analysis of grids}

Different structures of cantilever beams, shown in the above sections have been simulated in COMSOL multiphysics 5.2. Analytical modelling of those spiral cantilever beam has being carried out.

For solving purpose, three dimensional structure is considered as GRID structure as shown in figure 5. Eigen frequency is found by using COMSOL. In this analytical modelling of grid, natural frequency of the beam is obtained by stiffness matrix and mass matrix. A three beam structure is considered here for the sake of simplicity. 


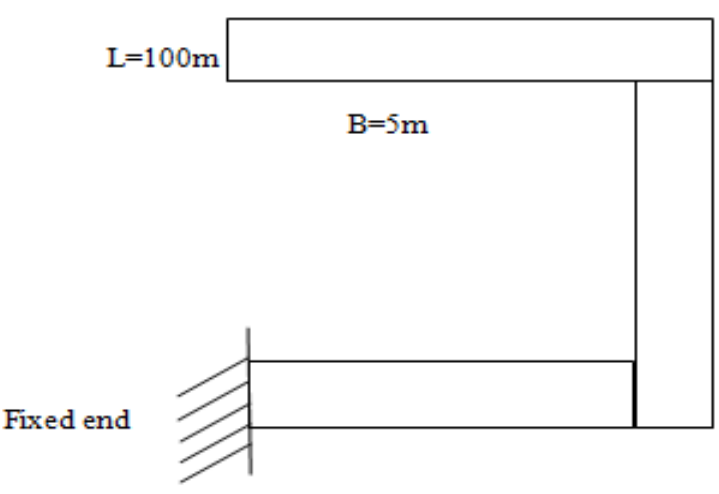

Fig. 5: Beam structure

Were $\mathrm{W}=$ Eigen values, $\mathrm{K}=$ stiffness matrix, $\mathrm{M}=$ mass matrix $\mathrm{K}\left[\mathrm{]}-\left(\mathrm{W}^{2}\right) * \mathrm{M}[]=0\right.$

The stiffness matrix for a grid element is given as:

$$
\left[\begin{array}{c}
p 1 \\
p 2 \\
p 3 \\
p 4 \\
p 5 \\
p 6
\end{array}\right]=\mathrm{EI} / \mathrm{L}^{3}\left[\begin{array}{cccccc}
J G L^{*} L / E I & & & \text { SYMMETRIC } & & \\
0 & 4 L^{*} L & & & & \\
0 & -6 L & 12 & & & \\
-J G L^{*} L / E I & 0 & 0 & J G L^{*} L / E I & & \\
0 & 2 L^{*} L & -6 L & 0 & 4 L^{*} L & \\
0 & 6 L & -12 & 0 & 6 L & 12
\end{array}\right]\left[\begin{array}{c}
\in 1 \\
\in 2 \\
\in 3 \\
\in 4 \\
\in 5 \\
\in 6
\end{array}\right]
$$

The mass matrix for the grid element is given as:-

$$
\left[\begin{array}{c}
p 1 \\
p 2 \\
p 3 \\
p 4 \\
p 5 \\
p 6
\end{array}\right]=\operatorname{ML} / 2\left[\begin{array}{cccccc}
140 I / A & & & \text { SYMMETRIC } & \\
0 & 4 L^{*} L & & & & \\
0 & 22 L & 156 & & & \\
70 I / A & 0 & 0 & 140 I / A & & \\
0 & -3 L^{*} L & -13 L & 0 & 4 L^{*} L & \\
0 & 13 L & 54 & 0 & -22 L & 156
\end{array}\right]\left[\begin{array}{c}
\in 1 \\
\in 2 \\
\in 3 \\
\in 4 \\
\in 5 \\
\in 6
\end{array}\right]
$$

Transformation of coordinates:

The stiffness matrix as well as mass matrix are in reference to the local system of coordinates.

Therefore, it is necessary to transform the reference of these matrices to the global system of coordinates before their assemblage in the corresponding matrices for the structure

$$
\operatorname{Tn}=\left[\begin{array}{cccccc}
\cos q & \sin q & 0 & 0 & 0 & 0 \\
-\sin q & \cos q & 0 & 0 & 0 & 0 \\
0 & 0 & 1 & 0 & 0 & 0 \\
0 & 0 & 0 & \cos q & \sin q & 0 \\
0 & 0 & 0 & -\sin q & \cos q & 0 \\
0 & 0 & 0 & 0 & 0 & 1
\end{array}\right]
$$

The material chosen for the cantilever beam is 'Polysilicon" The material properties of polysilicon are given in Table.1

Table 1: Material Properties of Polysilicon

\begin{tabular}{|l|l|}
\hline Young's modulus(E) & $160^{*} 10^{9} \mathrm{pa}$ \\
\hline Poisons ratio(w) & 0.22 \\
\hline & \\
\hline Density(d) & $2320 \mathrm{~kg} / \mathrm{m}^{3}$ \\
\hline L & $100 \mathrm{~m}$ \\
\hline b & $5 \mathrm{~m}$ \\
\hline h & $1 \mathrm{~m}$ \\
\hline Modulus of rigidity $(\mathrm{mps})(\mathrm{G})$ & $111.11^{*} 10^{9} \mathrm{~Pa}$ \\
$\mathrm{E}=\mathrm{G}(1+2 \mathrm{w})$ & \\
$160^{*} 10^{9=} \mathrm{G}(1+2 * 0.22)$ & \\
\hline $\mathrm{J}=$ polar moment of inertia $\left(\mathrm{mm}^{4}\right)$ & $2.5\left(\mathrm{~m}^{4}\right)$ \\
$\mathrm{J}=\mathrm{I}_{\mathrm{xx}}+\mathrm{Iyy}$ & \\
$(\mathrm{bh} / 12)+\left(\mathrm{b}^{3} \mathrm{~h} / 12\right)$ & \\
$(5 / 12)+(25 / 12)$ & \\
$0.41667+2.0833$ & \\
\hline
\end{tabular}

$\mathrm{T}=$ torque $(\mathrm{N}-\mathrm{mn})$ $\mathrm{q}=$ angular shift deflection

$\mathrm{I}_{\mathrm{m}}=$ mass polar moment of inertia $=\mathrm{bh}^{3} / 12=0.4166 \mathrm{~m}^{4}$

$\ddot{\mathrm{I}}=$ second moment of area or moment of inertia, (This value is equal to $\mathrm{J}$ )

$\mathrm{A}=\mathrm{L} * \mathrm{~b}=100 * 5=\mathbf{5 0 0 \mathbf { m } ^ { 2 }}$

After substituting the values we the Kn matrix and Mn matrix respectively, the $\mathrm{Kn}$ matrix and $\mathrm{Mn}$ matrix is given below:

$$
\mathrm{Kn}=\left[\begin{array}{cccccc}
2.75 * 10^{\wedge} 9 & & \multicolumn{5}{c}{\text { symmetric }} \\
0 & 2.6 * 10^{\wedge} 9 & & & & \\
0 & -0.04 * 10^{\wedge} 9 & 12 & & & \\
-2.75 * 10^{\wedge} 9 & 0 & 0 & 2.75 * 10^{\wedge} 9 & & \\
0 & 3.2 * 10^{\wedge} 9 & -0.04 * 10^{\wedge} 9 & 0 & 2.66 * 10^{\wedge} 9 & \\
0 & 0.04 * 10^{\wedge 9} & -12 & 0 & 0.04 * 10^{\wedge} 9 & 12
\end{array}\right]
$$

$$
\mathrm{Mn}=\left[\begin{array}{ccccc}
190133.3 & & & & \text { symmetric } \\
0 & x 1 & & & \\
0 & x 2 & 156 & & \\
95066.65 & 0 & 0 & 190133.3 & \\
0 & x 3 & -x 4 & 0 & x 1 \\
0 & x 4 & 54 & 0 & -x 2
\end{array}\right.
$$

$\mathrm{x} 1=1.0864 * 10^{10}, \mathrm{x} 2=597561800, \mathrm{x} 3=-8148570000$

$\mathrm{x} 4=353104700$

The obtained matrixes are in local directions, they should be transformed to global by using transformation matrix ' $T n$ '. By this we get three matrixes $\mathrm{K} 1, \mathrm{~K} 2, \mathrm{~K} 3$ \& $\mathrm{M} 1, \mathrm{M} 2, \mathrm{M} 3$.

Were, $\mathrm{T} 1, \mathrm{~T} 2, \mathrm{~T} 3$ are the transformation matrixes

$\mathrm{K} 1=[\mathrm{T} 1] \mathrm{T} * \mathrm{Kn} * \mathrm{~T} 1$;

$\mathrm{K} 2=[\mathrm{T} 2] \mathrm{T} * \mathrm{Kn} * \mathrm{~T} 2$;

$\mathrm{K} 3=[\mathrm{T} 3] \mathrm{T} * \mathrm{Kn} * \mathrm{~T} 3$;

$\mathrm{M} 1=[\mathrm{T} 1] \mathrm{T} * \mathrm{Mn} * \mathrm{~T} 1$

$\mathrm{M} 2=[\mathrm{T} 2] \mathrm{T} * \mathrm{Mn} * \mathrm{~T} 2$

$\mathrm{M} 3=[\mathrm{T} 3] \mathrm{T} * \mathrm{Mn} * \mathrm{~T} 3$;

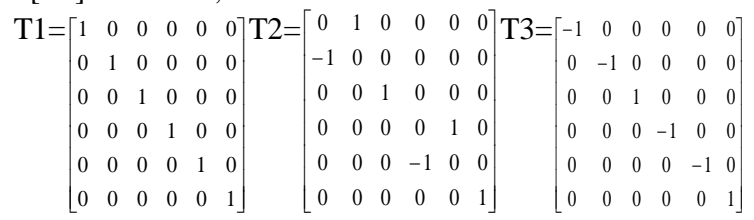

By using T1, T2, T3 the values of K1, K2, K3 \& M1, M2, and M3 are obtained.

By adding the values of $\mathrm{K} 1, \mathrm{~K} 2, \mathrm{~K} 3$, matrix $\mathrm{K}$ as $9 \mathrm{X} 9$ matrix is obtained and by adding the values of M1, M2 and M3 .

M matrix as 9X9 is obtained

$$
\begin{aligned}
& \mathrm{K}=10^{9 *}\left[\begin{array}{cccccccccc}
5.4 & & & \multicolumn{7}{c}{\text { symmetric }} \\
0 & 5.4 & & & & & & & \\
0.04 & 0.04 & 0.0 & & & & & & \\
3.2 & 0 & 0.04 & 5.4 & & & & & \\
0 & -2.7 & 0 & 0 & 5.4 & & & & \\
-0.04 & 0 & 0 & -0.04 & 0.04 & 0 & & & \\
0 & 0 & 0 & -2.7 & 0 & 0 & 2.7 & & \\
0 & 0 & 0 & 0 & 3.2 & 0.04 & 0 & 2.6 & \\
0 & 0 & 0 & 0 & -0.04 & 0 & 0 & -0.04 & 0
\end{array}\right] \\
& \mathbf{M}=10^{10 *}\left[\begin{array}{ccccccccc}
1.08 & & & & & \text { symmetric } & & \\
0 & 1.69 & & & & & & & \\
-0.06 & -0.06 & 0 & & & & & & \\
-0.81 & 0 & 0.035 & 1.08 & & & & & \\
0 & 0 & 0 & 0 & 1.08 & & & & \\
-0.035 & 0 & 0 & 0.06 & -0.06 & 0 & & & \\
0 & 0 & 0 & 0 & 0 & 0 & 0 & & \\
0 & 0 & 0 & 0 & -0.81 & 0.035 & 0 & 1.08 \\
0 & 0 & 0 & 0 & -0.035 & 0 & 0 & 0.06 & 0
\end{array}\right]
\end{aligned}
$$




\section{Results and conclusion}

Ambient vibration energy scavenging structure is proposed and analyzed in this work. The spiral cantilevers are mechanically independent in series give a compact design within an area of $100 \mu \mathrm{mX} 100 \mu \mathrm{m}$. The electrode configuration is designed and structures of different number of beams within the same footprint was analyzed. With the moment caused by the large dimensions of the proof mass considered, an analytical model was developed when suffering random external acceleration. The intrinsic characteristics of the Spiral structure are explored by FEA simulation. The results show that the natural frequency of the spiral structure is comparatively reduced as the number of beams keeps on adding. Adding the proof mass significantly reduces the resonant frequency. But the challenge in the design is to optimise the structure for minimum displacement at the tip of vibration. This can be probably achieved by using a double cantilever beam. Thus matching the resonant frequency would be easier if spiral structures are utilized. This means the Spiral structure proposed in this work could be feasible for effective energy harvesting from ambient vibrations.

Analytical modelling or Mathematical modelling is done by using FEA Grid methods, and this is performed to three beam element grid structure and results are obtained. The same three beam structure analysis is done in COMSOL multyphysics 5.2 software shown in figure. 5 and results obtained are approximately close to results of analytical modelling

The Eigen values obtained from analytical modelling are mentioned in the respective Table 2

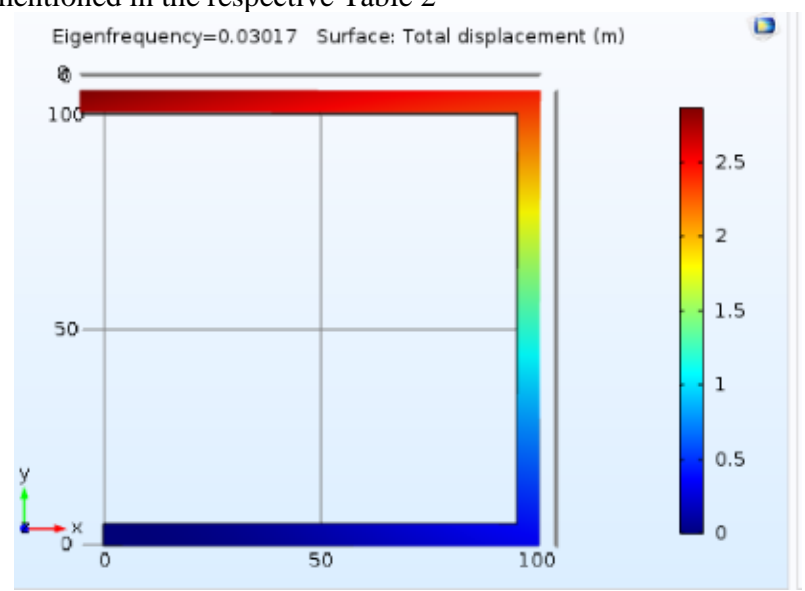

Fig. 6: Three beam cantilever structure

Table 2: Comparison between Theoretical and Practical Values

\begin{tabular}{|l|l|}
\hline $\begin{array}{l}\text { Eigen values obtained from } \\
\text { comsol multiphysics 5.2 }\end{array}$ & $\begin{array}{l}\text { Eigen values obtained from mathematical } \\
\text { modelling analysis of 3 beam structure }\end{array}$ \\
\hline 0.27262 & 0.2586 \\
\hline 0.52007 & 0.62017 \\
\hline 0.13001 & 0.353 \\
\hline 0.15782 & 0.6266 \\
\hline 0.03017 & 0.709 \\
\hline
\end{tabular}

\section{Future scope}

The structure proposed can be effectively utilized in modelling and fabrication of high efficient vibration energy harvesters' as compared to traditional cantilever structure. The work can be also be extended to double cantilever shape as the double cantilever structure is expected to further reduce the natural frequency of the structure.

\section{References}

[1] Bult K, Burstein A, Chang D, Dong M \& Fielding M, "A distributed, wireless MEMS technology for condition based maintenance", Proc. Integrated Monitoring, Diagnostics and
Failure Prevention Conference, Society of Machine Failure ProtectionTechnology (MPFT) (Mobile, AL, USA), (2006), pp.37380.

[2] Raghunathan V, Schurgers C, Park S \& Skrivastava MB, "Energyaware wireless microsensor networks", IEEE Signal Process. Mag. Vol.19, (2002), pp.40-50

[3] Enz CC, El-Hoiydi A, Decotignie JD \& Peiris V, "WiseNET: An ultralow-power wireless sensor network solution", IEEE Comput. Vol.37, (2004), pp.62-70

[4] Warneke B, Last M, Leibowitz B \& Pister KSJ, "Smart dust communicating with a cubic-millimeter computer", IEEE Comput., Vol.34, (2001), pp.44-51

[5] Raghunathan V, Schurgers C, Park S \& Srivastava MB, "Energyaware wireless microsensor networks", IEEE Signal processing magazine, Vol.19, No.2, (2002), pp.40-50.

[6] Roundy S, Wright PK \& Rabaye J, "A study of low level vibrations as a power source for wireless sensor nodes", Comput. Commun., Vol.26, (2003), pp.1131-1144.

[7] Starner T \& Paradiso JA, Human generated power for mobile electronics Low Power Electronics Design, ed C Piguet (Boca Raton, FL: CRC Press), (2004).

[8] von Buren T, Lukowicz P \& Tr"oster G, "Kinetic energy" powered computing-an experimental feasibility study", Proc. 7th IEEE Int. Symposium on Wearable Computers ISWC, (2003), pp.22-24.

[9] $\mathrm{Hu} \mathrm{H}$, Xue $\mathrm{H} \& \mathrm{Hu} \mathrm{Y,} \mathrm{"A} \mathrm{spiral-shaped} \mathrm{harvester} \mathrm{with} \mathrm{an}$ improved harvesting element and an adaptive storage circuit", IEEE transactions on ultrasonics, ferroelectrics, and frequency control, Vol.54, No.6,(2007).

[10] COMSOL Multiphysics tutorials. 